\title{
CONTROL OF SENSORLESS ELECTRICAL DRIVES OPERATING IN A HYBRID FLYING PLATFORM
}

\author{
Tomasz BINKOWSKI \\ Rzeszow University of Technology, ul. W. Pola 2, 35-959 Rzeszow, Poland \\ E-mail: tbinkow@prz.edu.pl
}

Received 27 January 2014; accepted 20 January 2015

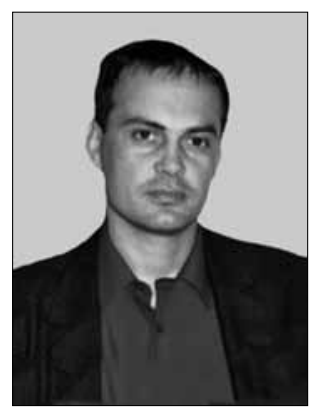

\begin{abstract}
Tomasz BINKOWSKI, PhD Eng.
Education: MSc (Eng) degree in electrical engineering, Rzeszow University of Technology, Faculty of Electrical and Computer Engineering, 1996; PhD degree in electrical engineering, Rzeszow University of Technology, Faculty of Electrical and Computer Engineering, specialization: power electronics, 2004.

Affiliations and functions: 2004 to present, researcher at Rzeszow University of Technology, Department of Power Electronics and Power Engineering.

Research interests: power electronics, control, hybrid drives.

Publications: author of 6 scientific articles and co-author of 15 conference papers.
\end{abstract}

Abstract. In the paper, the results of an investigation on the electrical drive of a hybrid flying platform (BAL) are presented. Due to restrictions on flying platforms it is necessary to use solutions with a limited number of sensors. For that purpose an investigation of two versions of electrical drives with a BLDC motor without a speed feedback has been conducted. The obtained results have been compared and conclusions regarding the practical use of the motor drive have been made. The investigations discussed in the paper concern only the proposed methods of speed estimation based on current measurement.

Keywords: electrical drive, hybrid flying platform, electric motor, new concept.

\section{Introduction}

Unmanned flying vehicles are being increasingly used in many areas, especially in military applications. Since the vehicles are unmanned they are cheaper to operate, they also have minimum dimensions which results in the reduction of fuel consumption. In many applications it is necessary to turn the machine on a quiet mode. In the case of an internal combustion engine it is a very hard requirement. Therefore, a development of a new concept of drive operating in the flying platform based on a hybrid solution is needed. In this paper a serial hybrid of an internal combustion engine with an electric motor with permanent magnets is presented. This solution seems to be universal due to the BLDC motor power density and efficiency. Also, due to the hybrid configuration the power of the internal combustion drive operating in the flying platform may be reduced since it is possible to support it during critical flight phases, e.g. climb phase.
The article deals in detail with the control of an electric motor with permanent magnets as a part of an unmanned flying platform. Problems and their solutions in terms of regulatory systems, modulation of control signals and the used inverter topology, are discussed in (Binkowski et at. 2011). Technical requirements of constructed platforms make the speed measurement, needed for the feedback, impossible. Apart from the sync issues of the drive shaft or engine start, the primary problem is reliable reproduction of the current rotational speed by measuring the inverter output current or direct current battery. BLDC motor current adjustment issues regarding how and where to measure it are described in many publications (Domoracki, Krykowski 2005; Concari et al. 2003; Pirog 2006; Dixon, Leal 2001).

Solutions presented in the article were elaborated based on knowledge of the mathematical model of a permanent magnet motor. The methodology of the study mainly focuses on the choice of a reference engine speed 
and its comparison with speed values that are reverse engineered in two ways and for various operating conditions. In order for the speed reference value to be reliable, the reference model was sampled with frequency many times greater than the sampling frequency of the predictive systems. In addition to the reference model, the known change of the load torque has been included. The reproducing speed systems were devoid of information about values of the load torque and speed reproduction relied exclusively on disturbed measuring current. A flowchart of the system using which the test was performed is illustrated in Figure 1.

Figure 1 the circuit consists of three functional blocks: S1as the reference model for permanent magnet engine, $\mathrm{S} 2$ - the speed reproduction system based on current measurement (version I), S3 - the speed reproduction system based on current measurement (version II based on D. G. Luenberger observation discussed in D. G. Luenberger (1971)).

Block S1 is a mathematical model of the BLDC motor with a power converter model. The inputs of $\mathrm{S} 1$ block are: battery voltage $\mathrm{u}(\mathrm{t})$, the moment of engine load $\mathrm{Ml}(\mathrm{t})$ and engine parameters, such as: $\mathrm{R}$ - resistance, $\mathrm{L}$ inductance, $\mathrm{J}$ - moment of inertia, Dp - friction coefficient, ke - electric constant, $\mathrm{km}$ - mechanical constant of engine, $\mathrm{Tm}$ - time period. The output values of S1 block are: engine speed and sum of the absolute values of the output currents of the power converter. Blocks S2 and S3 operate as prediction systems of engine speed. The engine speed is determined in S2 and S3 blocks on the basis of the value of the output current determined from S1 block. Moreover, the current is disturbed using an external noise generator. The inputs of S2 and S3 blocks are the value of the battery voltage $\mathrm{u}(\mathrm{t})$, measured output currents with noises of inverter and motor parameters

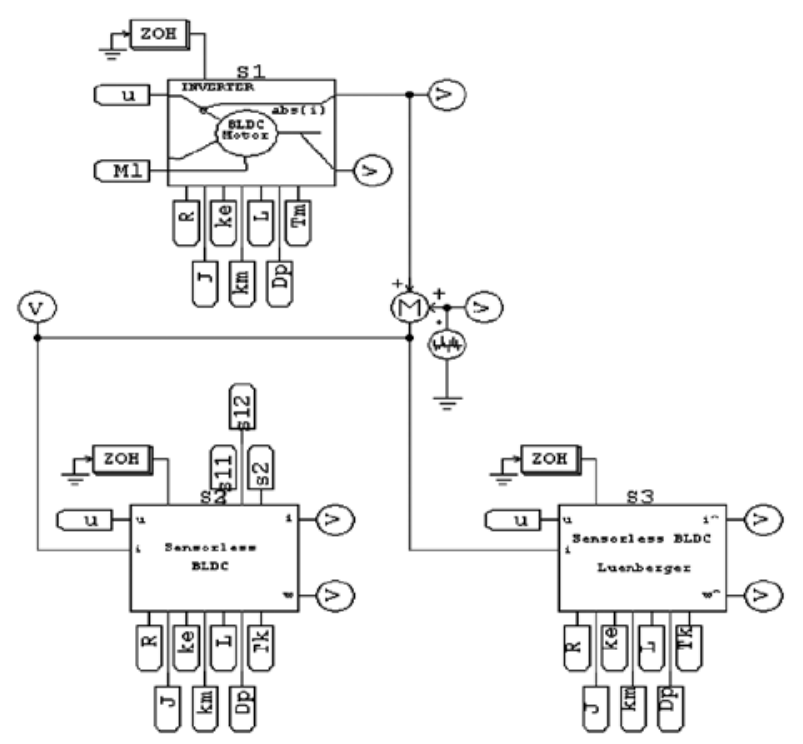

Fig. 1. A block diagram of the test circuit (such as for the unit S1, except Tk, which is several times smaller than the $\mathrm{Tm}$ ). The outputs are estimated value of the engine speed and the battery current. The value of the load torque is not known in S2 and S3 blocks. To block S2 values s2, s12 and s11 needed to determine normal distributions of the matrix in the estimation algorithm are also introduced.

\section{Mathematical model of the BLDC motor (S1 block)}

The design of the control system of the sensorless drive with a permanent magnet motor does not require a great accuracy of the mathematical model. The most important issue is to obtain the results from the model in a short time. Therefore, investigations of the sensorless drive with a BLDC motor were preceded by the mathematical peripheral model of the BLDC electric machine elaboration. Because of the simplicity of this model the digital form was developed using the FPGA Cyclone III EP3C40. In addition, the digital inverter model enabled to test our control strategy in real time, without laboratory tests.

Electrical circuit equation and the equation of mechanical binding engine torque with its speed are the basis for the mathematical model of the BLDC motor. In order to simplify the calculation of the engine model assumptions in the form of a linear motor windings' magnetic circuit without the phenomena of saturation and hysteresis and losses in iron were made. The constant resistance values and inductance windings were assumed too. Taking into account the formulated assumptions, the electrical circuit in the natural coordinate system can be represented by equation 1 below:

$$
u_{k}(t)=\mathrm{R} i_{k}(t)+\mathrm{L} \frac{d i_{k}(t)}{d t}+e_{k}(t),
$$

where $k=1,2,3$ is the ID of one of the three phases of the motor; $u_{k}$ - voltage of the $k$-th engine phase; R-resistance of a single phase motor; $i_{k}$ - current flowing in the $k$ phase of the engine; the $\mathrm{L}$ - inductance of motor; $e_{k}$ - electromotive induced in the $k$ phase.

The mechanical equation of BLDC motor is presented in equation 2 .

$$
\frac{d \omega(t)}{d t}=\frac{1}{\mathrm{~J}}\left(T_{e}(t)-T_{m}(t)\right)
$$

where $\omega$ is angular velocity; $J$ - moment of inertia; $T_{e}-$ electric torque; $T_{m}$ - torque of load.

In the case of the BLDC motor the induced electromotive force in each of the phases is shifted relative to others, passing an angle of $\frac{2}{3} \pi$, and, at the same time, the motor control must be carried out in such a way as to achieve a constant torque value. Therefore, the instant power delivered to the engine must be constant. 
The stability of the value of instant power at the trapezoidal wave can be obtained when individual phase currents flow at a constant value of electromotive force. The BLDC motor control is explained in many works (Domoracki, Krykowski 2005; Pirog 2006; Bose 2002). The flow of current during individual phases of the engine is closely linked with the state of the control process. Therefore, an electromotive can be described through the state of the control (Equation 3) and is easily made into a digital model. Engine torque is described in equation 4 .

$$
\begin{aligned}
& e_{k}(t)=k_{e} \omega(t) g_{k}(t) ; \\
& T_{e}(t)=k_{t} g_{k}(t) \sum_{k} i_{k}(t),
\end{aligned}
$$

where $k_{e}$ indicates voltage constant; $g_{k}$ - the function defining the electromotive force of $k$-phase which depends on a discrete state of inverter; $k_{t}$ - torque constant; $i_{k}-$ current of $k$-phase.

The digital engine model assumes that the waveforms of currents and voltages are determined depending on one of the six states that the inverter is in. The final value of the previous state is the initial state value at the next. The function of the shaping process of induced electromotive force in every stage is provided in equation 5 below:

$$
g_{k}(t)=\left\{\begin{array}{cl}
1, & \text { for } c_{k}=0 \\
-1, & \text { for } c_{k}=1 \\
12 f t-1, & \text { for } c_{k}=2 \\
-12 f t+1, & \text { for } c_{k}=3
\end{array},\right.
$$

where $f$ is the frequency of output waveform; $c_{k}$ - the value that depends on the status of the inverter in accordance with table; $t$ - time within a specified range depending on the status of the inverter. Using this concept it is assumed that the status code for the first character from the left encodes the state of phase 1, the middle character encodes phase 2 status, and a sign on the right side of the status refers to phase 3 . The values used for coding are as follows: a value of 1 means a conductive unit connector connected to the positive pole of the battery, a value of 0 indicates a conductive connector connected to the negative pole of the battery, and character “-" refers to a disabled branch in the inverter (Table).

Table. Inverter status codes

\begin{tabular}{cccc}
\hline STATE & $c_{1}$ & $c_{2}$ & $c_{3}$ \\
\hline $10-$ & 0 & 1 & 3 \\
\hline $01-$ & 1 & 0 & 2 \\
\hline $1-0$ & 0 & 2 & 1 \\
\hline $0-1$ & 1 & 3 & 0 \\
\hline-10 & 3 & 0 & 1 \\
\hline-01 & 2 & 1 & 0 \\
\hline
\end{tabular}

\section{BLDC motor speed reconstruction-version I (block S2)}

Based on equations 1 and 2 in a two-dimensional state space the engine description was elaborated as a state vector in the form of angular velocity and the sum of the absolute values of currents. As a force vector the vector that represents the value of the battery voltage was adopted. After digitization and linearization of the engine model taking into account the measurement disturbance $v(k)$ and the vector of state variables' disturbance $w(k)$ the general form of the mathematical model of the BLDC motor is illustrated by the equations below (6):

$\left\{\begin{array}{l}x(k+1)=A(k) x(k)+B(k) u(k)+w(k) \\ y(k)=C(k) x(k)+D(k) u(k)+v(k)\end{array}\right.$.

For the definition of equations of block S2 the following assumptions are made:

- $w(k), v(k)$ are uncorrelated matrices;

- $N\left(0, \sigma_{1}\right), N\left(0, \sigma_{2}\right)$ - distributions of normal matrices $w(k)$ and $v(k)$;

- $E[w(k)]=0, E[v(k)]=0$ - expected values of matrices $w(k)$ and $v(k)$;

- $E\left\{w(k) w^{T}(k)\right\}=Q(k), E\left\{v(k) v^{T}(k)\right\}=R(k)$ - covariance matrices $w(k)$ and $v(k)$;

- $x_{0}$ - the vector random variables with normal distribution;

- $E\left[x_{0}\right]=x_{0}-$ the expected value of vector $x_{0}$;

- $E\left[\begin{array}{ll}x_{0} & x_{0}^{T}\end{array}\right]=P_{0}$ - covariance matrix of vector $x_{0}$;

- $\hat{x}(0 /-1)=x_{0}-$ the initial value of the estimated state vector;

- $\sum(0 /-1)=P_{0}-$ the initial value of the covariance of prediction error.

The estimation of the state vector is carried out using two equations (7) which are almost identical for most observers. The difference in this case relates to the methods for determining the boost matrix (8). The boost matrix is the time-variant matrix because its elements depend on the time and statistical properties of disturbances:

$$
\begin{aligned}
& \left\{\begin{array}{l}
\hat{x}(k)=\hat{x}(k / k-1)+K(k)(y(k)-C(k) \hat{x}(k / k-1)) \\
y(k)=C(k) x(k)+D(k) u(k)+v(k)
\end{array},\right. \\
& K(k)=\sum(k / k-1) C^{T}(k) . \\
& \left(C(k) \sum(k / k-1) C^{T}(k)+Q(k)^{-1}\right),
\end{aligned}
$$

where $\sum(k / k-1)$ is the prediction error covariance at the $k$ time depends on the covariance estimation error $\left(\sum(k)\right)$ at time $k ; \hat{x}(k / k-1)$ - an estimate of the state vector obtained through the prediction process. 


\section{BLDC motor speed reconstruction-version II (block S3)}

The second version of the speed reconstruction system of the BLDC motor is based on the measurement of currents which is elaborated based on a typical, classic state observer proposed by D. G. Luenberger (1971):

$$
\left\{\begin{array}{l}
x(k+1)=A(k) x(k)+B(k) u(k) \\
y(k)=C(k) x(k)+D(k) u(k)
\end{array} .\right.
$$

Assuming that it is possible to describe the motor by state equations (9), the engine speed can be estimated by adding the correction matrix, created by the $H$ matrix multiplied by error terms calculated in the previous step, to the state equation.

Elements of matrix $H$ are chosen so that the transitional process of the observer is six times shorter than the transitional processes occurring in the BLDC motor. The digital calculation carried out in order to calculate speed and current are shown in Figure 2.

\section{Selected results of the investigation}

The research data of the designed and produced BLDC motor have been assumed, using these specifications: $U=24-29.6 \mathrm{~V}, n=8000 \mathrm{rpm}, J=0.004 \mathrm{~kg} / \mathrm{m}^{2}$, $L=386 \mu \mathrm{H}, R=0.027 \Omega, k_{e}=5.27 \mathrm{~V} / 1000 \mathrm{rpm}, k_{t}=$ 0.0391 . During the research the typical situations that
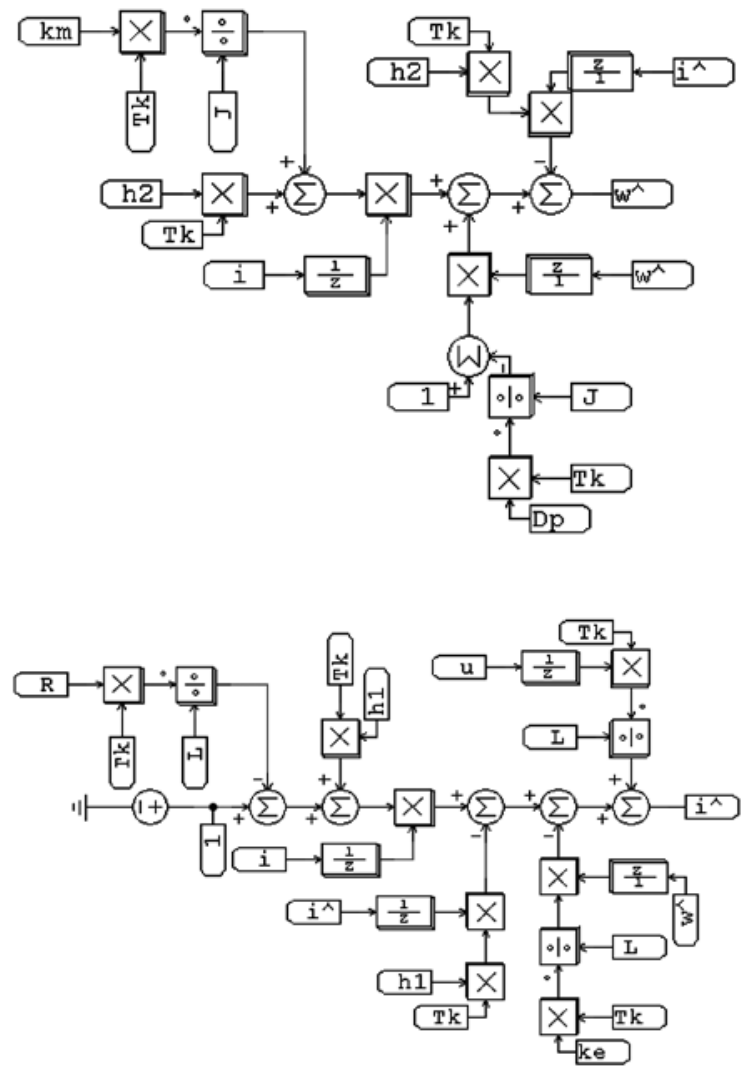

Fig. 2. Diagrams illustrating the speed and sum of the calculation of currents may occur during the operation of the flying platform, consisting of assisting the climbing process, silent mode flight, battery consumption, external disorders affecting on moment load, as well as the distortion of measurements were explored. The article contains some cases for illustration due to the large number of results (Figs 3-6).

A. The state without load
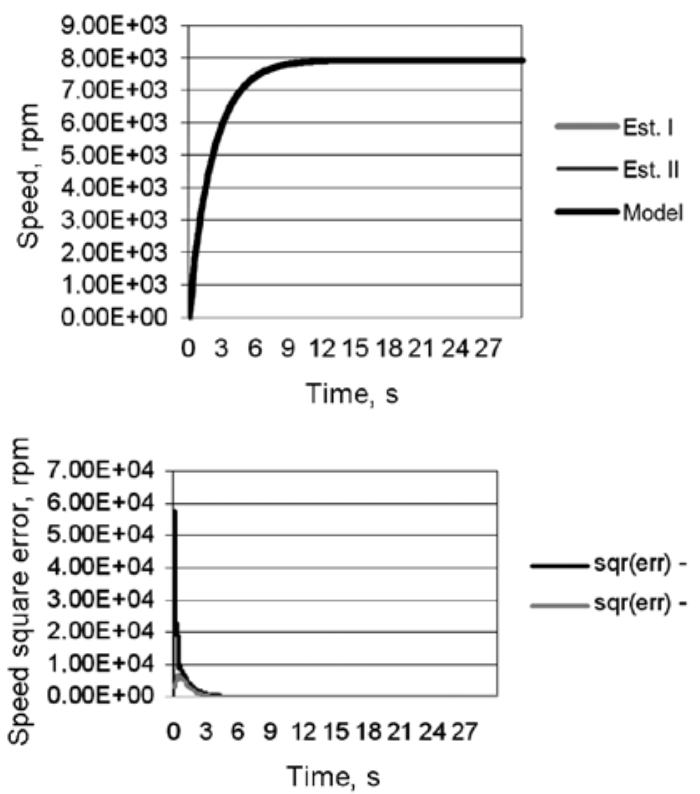

Fig. 3. Drive speed: model as the relation model, Est. I estimation by system I, Est. II - estimation by system II; sqr(err)-I, sqr(err)-II - related square error of speed for estimation in system I/II respectively

B. The step change of load (attachment of the BLDC drive to a combustion engine)
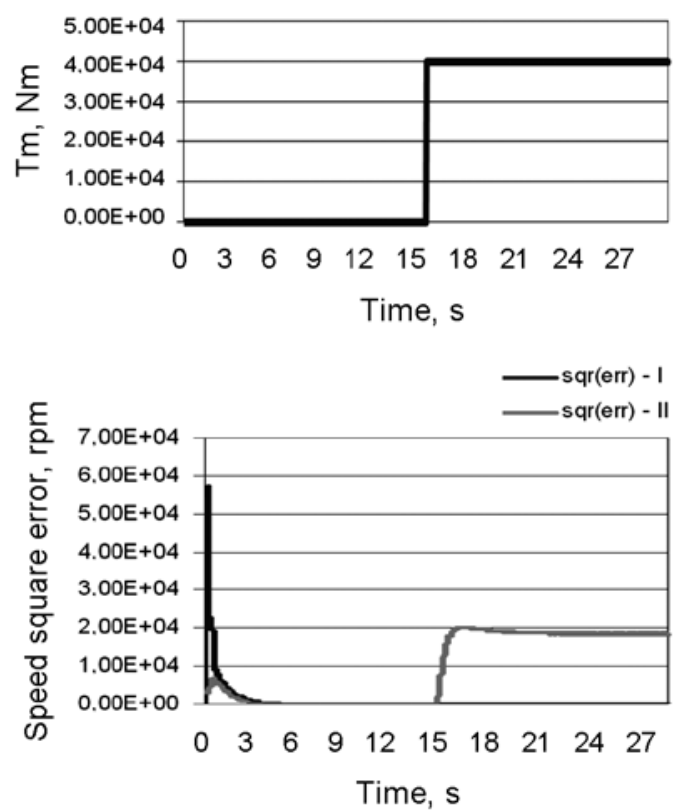

Fig. 4. Load torque $T_{m}$ and related square error (sqr(err)) of speed for estimation in system I/II respectively during the attachment process of the BLDC drive to the engine 


\section{Linear change of the load (launching hybrid)}
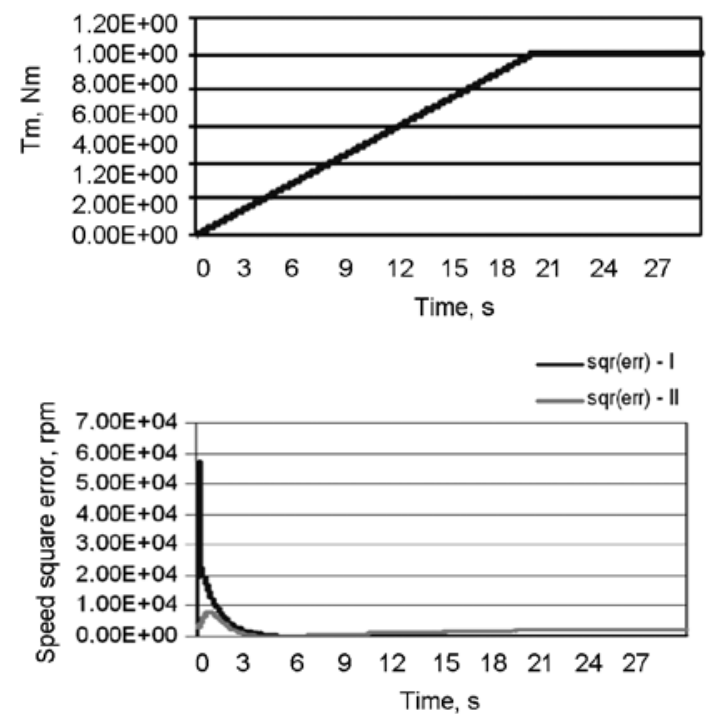

Fig. 5. Load torque $T_{m}$ and related square error (sqr(err)) of speed for estimation in system I/II respectively during launching hybrid process with the BLDC drive
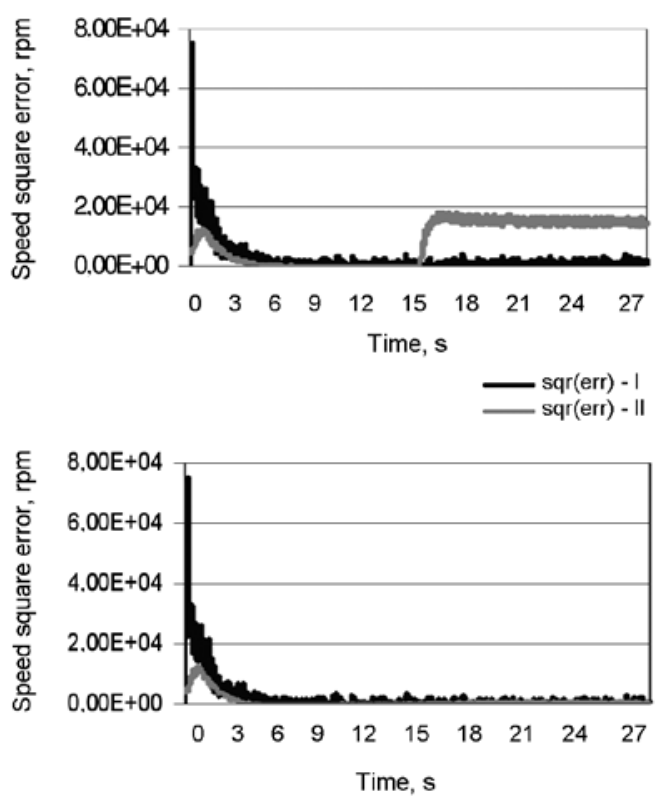

Fig. 6. Related square error (sqr(err)) of speed for estimation in system I/II during the attachment process of the BLDC drive to the engine and launching hybrid process with the BLDC drive respectively - high level of measurement noise

\section{Conclusions}

Studies clearly indicate the advantage of reproducing drive speed with a BLDC motor using the algorithm of version I type. This algorithm is characterized by a significantly smaller estimation error in relation to the estimation error of the algorithm in version II in the area of dynamic operation. In the case of measurement of currents with a high noise algorithm in version II, it is more prone to move them to an estimated value. In addition, the estimation values are affected by the change of object parameters. The effect of the parameter object changes and the noise level for variant I can be corrected by the proper selection of the appropriate normal distributions for matrix $w(k)$ and $v(k)$. Selection of coefficients characterizing the normal distribution should be dependent on the accuracy of the mathematical model and accuracy of obtaining consensus on establishing the range of a hybrid system operation. In the case of variant II a level of disruption and the impact of object parameter changes shall be taken into account by the proper selection of appropriate gain values of the observer. Tuning issues of observer systems are complex and are not the subject of the article.

\section{References}

Binkowski, T.; Buczek, K.; Sobczynski, D. 2011. Control of BLDC motor operating as part of a hybrid flying platform, Przeglad Elektrotechniczy [Electrical Review] 8 (in Polish).

Bose, B. K. 2002 Modern Power Electronics and AC Drives. Prentice Hall PTR.

Concari, C.; Franceschini, G.; Raboni, S. 2003. Single Current Sensing for Brushless DC Derives: A Fully Digital Solution. EPE, Toulouse.

Dixon, J.; Leal, I. 2001. Current control strategy for brushless DC motors based on a common DC signal, IEEE Transactions on Power Electronics 17(2): 232-240. http://dx.doi.org/10.1109/63.988834

Domoracki, A.; Krykowski, K. 2005. BLDC Motors - classic control methods, Zeszyty Problemowe - Maszyny Elektrycz$n e$ [Transactions - Electrical Machines] (72) (in Polish).

Luenberger, D. G. 1971. An introduction to observers, IEEE Transactions on Automatic Control 16(6): 596-602. http://dx.doi.org/10.1109/TAC.1971.1099826

Pirog, S. 2006. Power Electronics: Circuits with Self-switching and Hard-switching. AGH Uczelniane Wydawnictwa Naukowo-Dydaktyczne (in Polish). 